\title{
A Reminder of Treatments Past
}

\author{
Kamran Hassan a, b, Saba Lahsaei ${ }^{\mathrm{a}}$, Anna Beyer ${ }^{\mathrm{a}}$, \\ Richard H. Hongo ${ }^{\mathrm{a}}$
}

\begin{abstract}
The Wolff-Parkinson-White (WPW) syndrome was initially described in 1930 as a clinical syndrome of paroxysmal tachycardias in healthy young people that demonstrated "bundle-branch block and short P-R interval". These tachycardias were eventually determined to be macro-reentrant arrhythmias utilizing accessory pathways. Surgical approaches for transecting these accessory pathways were being developed as early as the 1960s. Since 1984, catheter ablation has developed into a safe and highly effective therapy option, and the treatment of choice for WPW syndrome today. We present a unique case of a patient with previous surgically treated WPW syndrome that serves as a reminder of treatments past and caution for current therapies. A 54-year-old man with a remote history of WPW syndrome, treated in 1989 with surgical cryoablation of a left ventricular free wall accessory pathway, now presented with an hour of sustained palpitations after a triathlon practice session. He was found to have ventricular tachycardia at a rate of $180 \mathrm{bpm}$ on EKG requiring external cardioversion. The troponin I level peaked at $2.59 \mathrm{ng} / \mathrm{mL}$. Left heart catheterization revealed a peculiar truncated $100 \%$ occlusion of the proximal circumflex artery with extensive bridging collaterals to the mid circumflex artery, and also confirmed an infero-basal aneurysm. The cause of the ventricular tachycardia (VT) was deemed non-ischemic and he was referred for electrophysiology study. 3D electroanatomic mapping of the left ventricular (LV) endocardial surface was performed, revealing a discrete posterior wall scar. Reentrant VT was repeatedly induced utilizing a zone of slow conduction along the border zone of the scar. Radiofrequency ablation of Purkinje potentials on the LV septum, running alongside the scar border zone, eliminated the VT. The entire endocardial surface of the postero-basal scar was electrically silenced to prevent further VT events. Typically, during the surgical approach of WPW, a wide endocardial (rarely an epicardial) incision was made along the atrial surface of the mitral valve annulus, dissecting deeply and caudally until ventricular myocardium was exposed. Despite the close proximity of the circumflex artery to the epicar-
\end{abstract}

Manuscript accepted for publication April 19, 2016

${ }^{a}$ California Pacific Medical Center, San Francisco, CA, USA

${ }^{b}$ Corresponding Author: Kamran Hassan, CPMC, 2333 Buchanan Street, San

Francisco, CA 94115, USA. Email: Kamranhassan84@gmail.com

doi: http://dx.doi.org/10.14740/jmc2488w dial incision line during these surgeries, there are only rare reports of coronary artery complications, generally considered to be either catheter-induced dissections or embolic events. The development of a network of extensive bridging collaterals in our patient supports progressive stenosis of the left circumflex artery from cryoablation thermic injury, leading to scar formation and VT years later. In conclusion, this case reminds us that current ablative therapies are still relatively new, and long-term follow-ups for delayed complications remain a necessity.

Keywords: Arrhythmia; WPW; Catheter ablation

\section{Introduction}

Wolff-Parkinson-White (WPW) syndrome was initially described in 1930 as a clinical syndrome of paroxysmal tachycardias in healthy young people that demonstrated "bundlebranch block and short P-R interval". It was not till the 1940s that extranodal accessory pathways were discovered, and not till the 1950s that accessory pathways were used to explain the characteristic ECG pattern we know of today as "delta waves". The tachycardias were eventually determined to be macroreentrant arrhythmias utilizing accessory pathways. It was also recognized that the occurrence of atrial fibrillation in the setting of WPW syndrome could result in cardiac arrest. Because of the potentially life-threatening nature of the syndrome, surgical approaches for transecting accessory pathways were being developed as early as the 1960 s. Since the first description of catheter ablation of an accessory pathway by Morady and Scheinman in 1984 [1], catheter ablation has developed into a safe and highly effective therapy option, and the treatment of choice for WPW syndrome today.

\section{Case Report}

A 54-year-old man with a remote history of WPW syndrome, treated in 1989 with surgical cryoablation of a left ventricular free wall accessory pathway, now presented with an hour of sustained palpitations after a triathlon practice session. $\mathrm{He}$ was found to have a wide complex tachycardia at a rate of 180 bpm (Fig. 1). Hemodynamic instability prompted external 


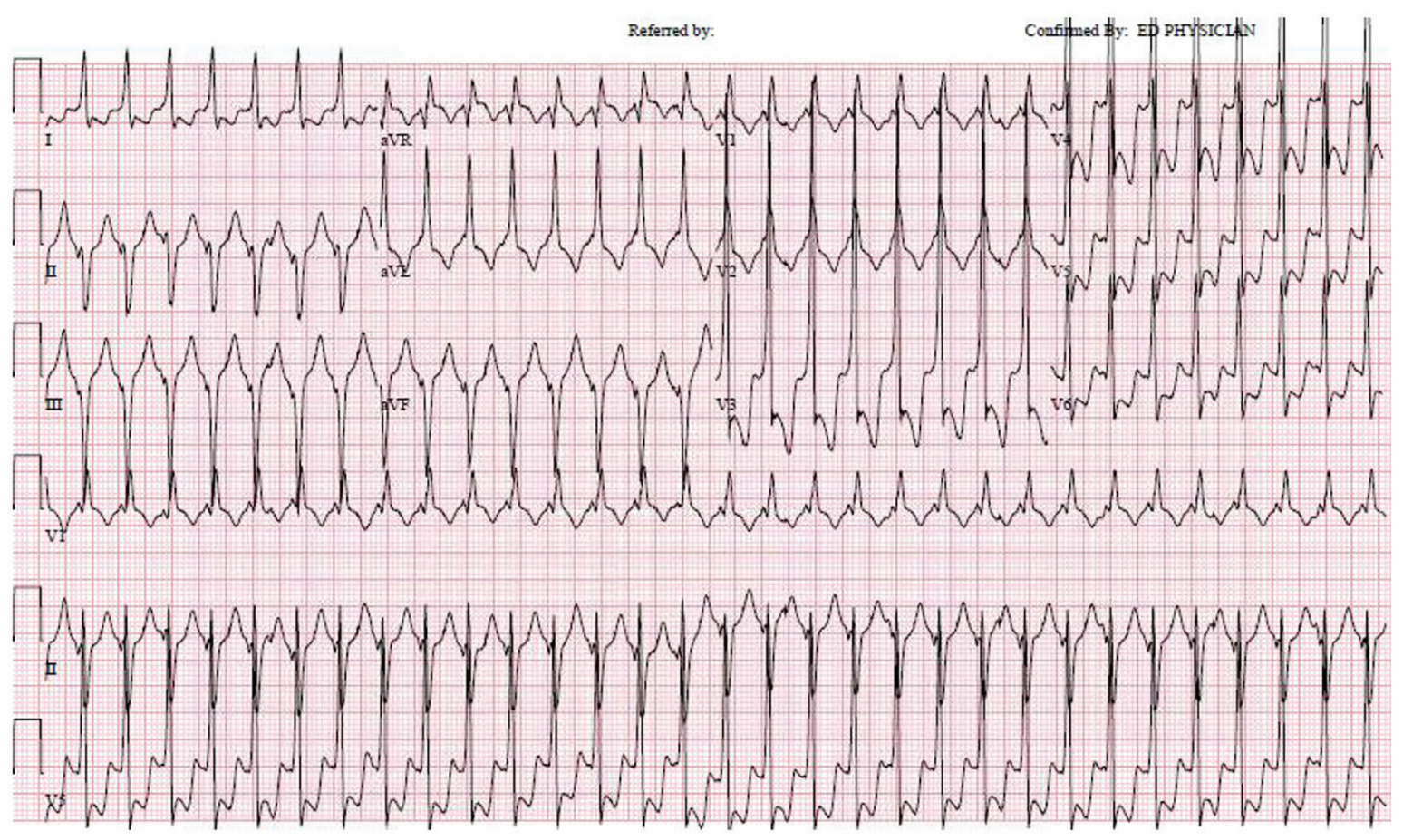

Figure 1. Regular wide complex tachycardia at $180 \mathrm{bpm}$, with a relatively narrow QRS duration of $120 \mathrm{bpm}$. The RBBB pattern with left axis deviation is typical for a fascicular VT originating from the LV septum.

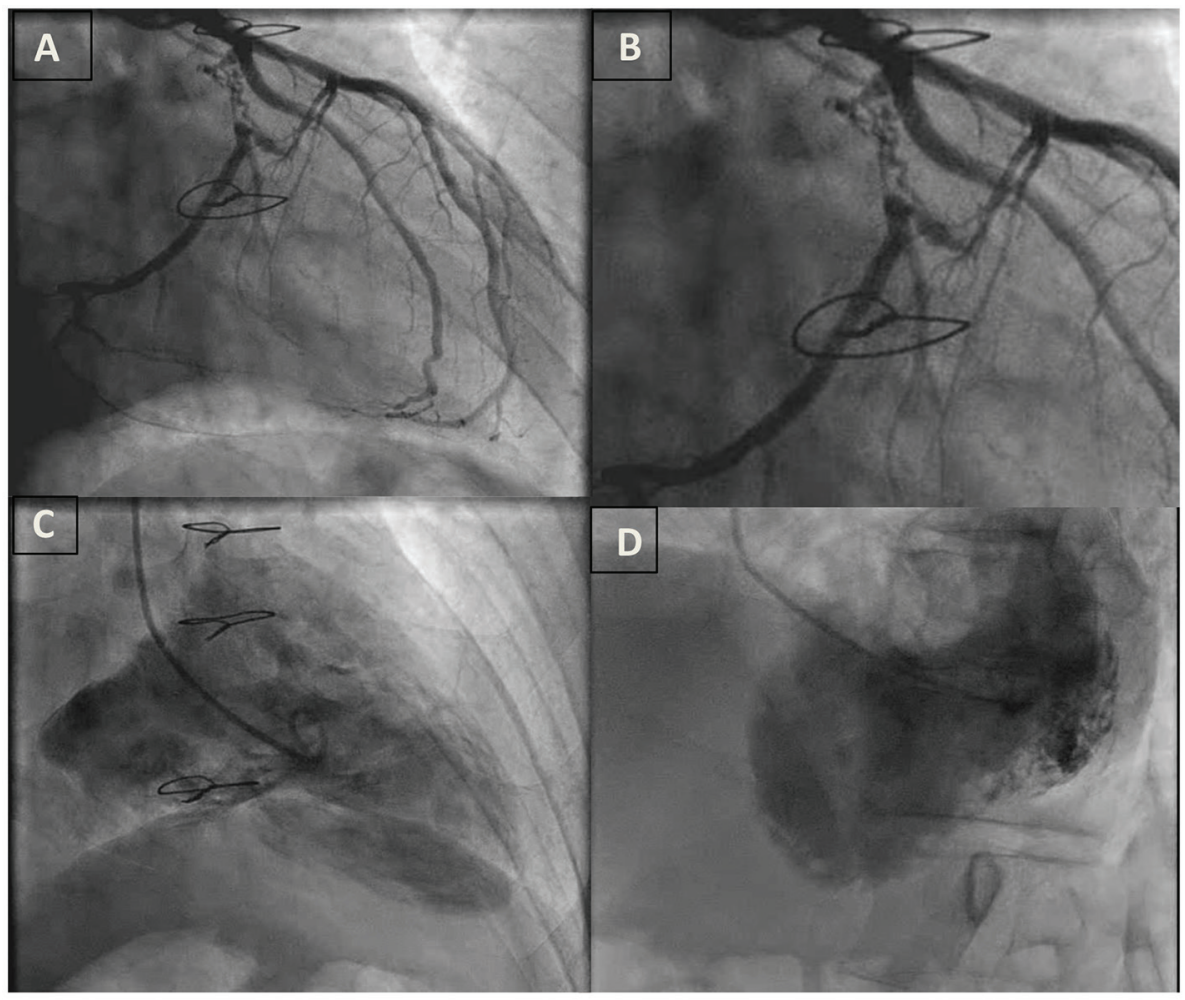

Figure 2. (A) Proximal circumflex artery occlusion with microchannels and left-to-left collateral circulation. (B) Close up view of A. (C) RAO view of left ventriculogram with an infero-basal aneurysm. (D) LAO view of left ventriculogram with an infero-basal aneurysm. 


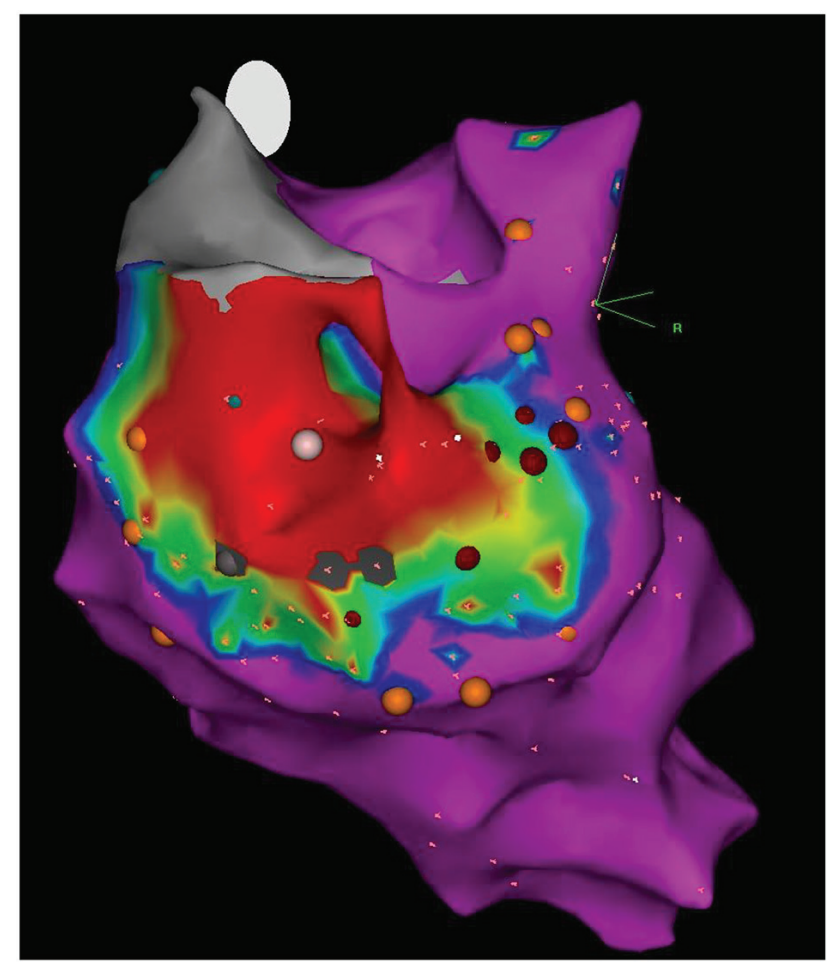

Figure 3. 3D electroanatomic voltage map of the endocardial surface of the left ventricle, posterior view. Discrete posterior wall scar (bipolar, $<0.5 \mathrm{mV}$; region of red) extends from the base to mid ventricle.

cardioversion and normal sinus rhythm was restored with a single attempt. The ECG in sinus rhythm did not demonstrate ischemic changes or delta waves. Transthoracic echocardiogram revealed an infero-basal wall motion abnormality that suggested aneurysmal dilatation. The troponin I level peaked at $2.59 \mathrm{ng} / \mathrm{mL}$. Left heart catheterization revealed a peculiar truncated $100 \%$ occlusion of the proximal circumflex artery with extensive bridging collaterals to the mid circumflex artery, and also confirmed an infero-basal aneurysm (Fig. 2). The cause of the ventricular tachycardia (VT) was deemed non-ischemic and he was referred for electrophysiology study.

3D electroanatomic mapping of the endocardial surface of the left ventricle (LV) was performed, and a discrete posterior wall scar that extended from the mitral valve annulus to the mid ventricle was defined (Fig. 3). Within the scar, multiple sites with fragmented slow continuous electrograms were discovered, confirming the presence of numerous channels of conduction within scar that could facilitate reentrant VT. Monomorphic VT consistent with the clinical VT was induced, and the exit site of this VT circuit was localized to the septal border of the postero-basal scar. The relatively narrow VT (QRS duration of $120 \mathrm{~ms}$ ) was explained by the presence of the branches of the posterior fascicle in proximity to the exit site (Fig. 4). It was postulated that the VT was a variant of fascicular VT that utilized branches of the posterior fascicle as the retrograde rapidly conducting limb of the reentry tachycardia, and a region of slow conduction between Purkinje fibers as the antegrade slowly conducting limb. In

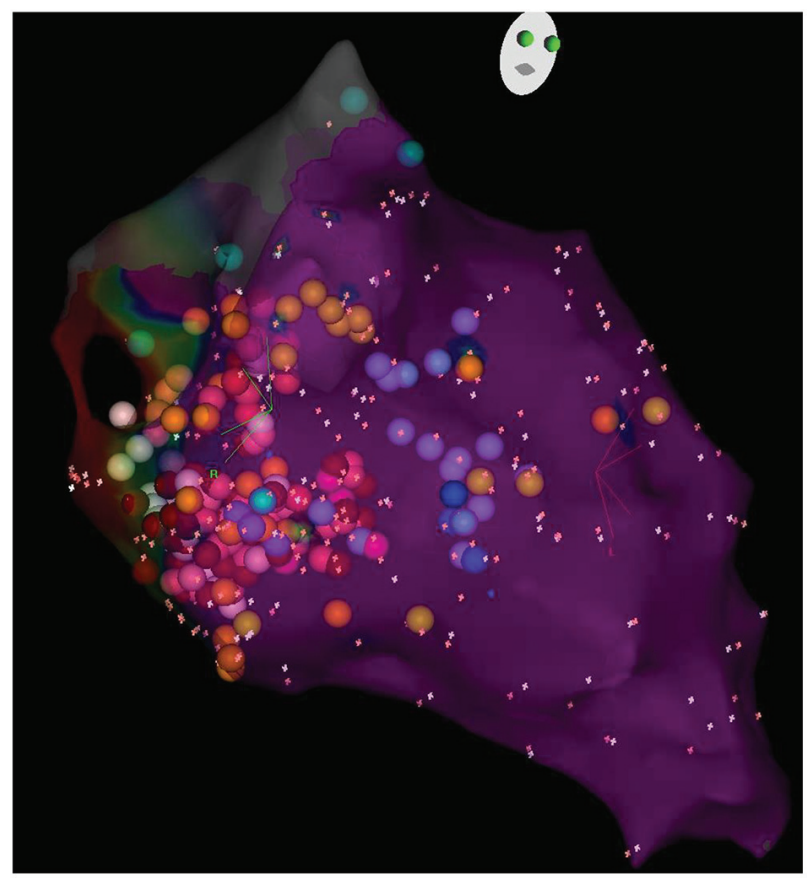

Figure 4. 3D electroanatomic map of the left ventricular septum, RAO caudal view. The sites of Purkinje potentials are marked (light purple dots) and outline the course of the branches of the left posterior fascicle.

this case, delayed conduction through the postero-basal scar was likely acting as the antegrade limb. In support of this postulated mechanism, radiofrequency ablation of Purkinje potentials along the septal border of the postero-basal scar made the VT non-inducible. The entire endocardial surface of the postero-basal scar was electrically silenced to prevent further VT events.

\section{Discussion}

The first surgical localization of a WPW syndrome accessory pathway was reported in 1967. Actual surgical transactions of the accessory pathways were initially met with only limited success, largely due to the limitations in electophysiological testing at the time of surgery. As mapping techniques improved and epicardial cryoablation became utilized [2], surgical treatment of WPW syndrome became predictably successful [3]. The first transvenous approach treating WPW in 1984 utilized high energy direct current countershocks [1]. With the development of radiofrequency catheter ablation, by the 1990s, catheter ablation had become the primary option for patients with WPW syndrome with drug-refractory recurrent tachycardias. With success rates consistently $>95 \%$ and complication rates $<5 \%$ [4], radiofrequency catheter ablation is the treatment of choice for treatment of WPW syndrome, and it is increasingly rare for patients to be managed on long-term antiarrhythmic drugs.

During the surgical approach, a wide endocardial incision was typically made along the atrial surface of the mitral 
valve annulus, dissecting deeply and caudally until ventricular myocardium was exposed. If necessary, an epicardial incision was added directly above the coronary sinus to facilitate epicardial cryoablation. The course of the circumflex artery is epicardial, within the atrio-ventricular groove, running parallel to the coronary sinus and the mitral annulus. Despite the close proximity of the circumflex artery to the incisions and ablations performed during these surgeries, there are only rare reports of coronary artery complications, generally considered to be either catheter-induced dissections or embolic events [5].

In our case, the development of a network of extensive bridging collaterals supports a process that more likely developed over time, and not with an acute dissection or embolic event. This would raise the possibility of thermic injury to the proximal left circumflex artery from the cryoablation that resulted in progressive stenosis and eventual 100\% occlusion. Endocardial ablations performed in the last 10 - 20 years frequently utilize high-power delivery catheters that can make deeper lesions. Thermic injury to the circumflex artery is well documented even from an endocardial approach, although usually subclinical [6]. This case raises concern that given more time, subclinical injuries of coronary arteries from the thermic effect of ablation may manifest years later as myocardial injury and even life-threatening ventricular tachyarrhythmias.

In conclusion, this case is a reminder of treatments from the past and it is a chance to review the early history of electrophysiology that in many ways revolved around the treatment of WPW syndrome. While it helps us appreciate how far our treatments have come in the management of arrhythmias, it reminds us that current ablative therapies are still relatively new, and long-term follow-ups for delayed complications are still a necessity.

\section{Funding}

None.

\section{Conflicts of Interest}

None.

\section{References}

1. Morady F, Scheinman MM. Transvenous catheter ablation of a posteroseptal accessory pathway in a patient with the Wolff-Parkinson-White syndrome. N Engl J Med. 1984;310(11):705-707.

2. Klein GJ, Guiraudon GM, Perkins DG, Jones DL, Yee $\mathrm{R}$, Jarvis E. Surgical correction of the Wolff-ParkinsonWhite syndrome in the closed heart using cryosurgery: a simplified approach. J Am Coll Cardiol. 1984;3(2 Pt 1):405-409.

3. Cox JL. Cardiac surgery for arrhythmias. Pacing Clin Electrophysiol. 2004;27(2):266-282.

4. Thakur RK, Klein GJ, Yee R. Radiofrequency catheter ablation in patients with Wolff-Parkinson-White syndrome. CMAJ. 1994;151(6):771-776.

5. Doguet F, Le Guillou V, Litzler PY, Bouchart F, NafehBizet C, Cribier A, Bessou JP. Coronary artery dissection after surgical cryoablation procedure. Ann Thorac Surg. 2009;87(6): 1946-1948.

6. Wong KC, Lim C, Sadarmin PP, Jones M, Qureshi N, De Bono J, Rajappan K, et al. High incidence of acute subclinical circumflex artery 'injury' following mitral isthmus ablation. Eur Heart J. 2011;32(15):1881-1890. 